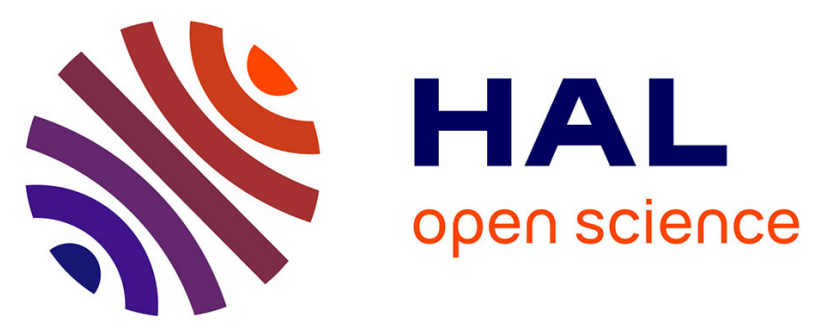

\title{
Harnessing polymer grafting to control the shape of plasmonic nanoparticles
}

Ying Zhou, Liting Yan, Tanmoy Maji, Gaëtan Lévêque, Manos Gkikas, George Fytas

\section{- To cite this version:}

Ying Zhou, Liting Yan, Tanmoy Maji, Gaëtan Lévêque, Manos Gkikas, et al.. Harnessing polymer grafting to control the shape of plasmonic nanoparticles. Journal of Applied Physics, 2020, 127 (7), pp.074302. 10.1063/1.5140459 . hal-03142228

\section{HAL Id: hal-03142228 \\ https://hal.science/hal-03142228}

Submitted on 27 Aug 2021

HAL is a multi-disciplinary open access archive for the deposit and dissemination of scientific research documents, whether they are published or not. The documents may come from teaching and research institutions in France or abroad, or from public or private research centers.
L'archive ouverte pluridisciplinaire HAL, est destinée au dépôt et à la diffusion de documents scientifiques de niveau recherche, publiés ou non, émanant des établissements d'enseignement et de recherche français ou étrangers, des laboratoires publics ou privés. 


\title{
Harnessing polymer grafting to control the shape of plasmonic nanoparticles
}

\author{
Cite as: J. Appl. Phys. 127, 074302 (2020); doi: 10.1063/1.5140459 \\ Submitted: 3 December 2019 . Accepted: 3 February 2020 . \\ Published Online: 18 February 2020
}

Ying Zhou, ${ }^{7}$ Liting Yan, ${ }^{1,2}$ Tanmoy Maji, ${ }^{3}$ Gaëtan Lévêque, ${ }_{4}^{4}$ Manos Gkikas, ${ }^{3, a)}$ (D) and George Fytas $\left.2, a\right)$

\author{
AFFILIATIONS \\ ${ }^{1}$ School of Chemistry and Chemical Engineering, Shanxi University, Taiyuan 030006, China \\ ${ }^{2}$ Max Planck Institute for Polymer Research, Ackermannweg 10, 55128 Mainz, Germany \\ ${ }^{3}$ Department of Chemistry, University of Massachusetts Lowell, Lowell, Massachusetts 01854, USA \\ ${ }^{4}$ Institut d'Électronique, de Microélectronique et de Nanotechnologie (IEMN), UMR-CNRS 8520, UFR de Physique, \\ Université de Lille 1, 59655 Villeneuve d'Ascq, France
}

Note: This paper is part of the Special Topic on: Polymer-Grafted Nanoparticles.

${ }^{a}$ Authors to whom correspondence should be addressed: manos_gkikas@uml.edu and fytas@mpip-mainz.mpg.de

\begin{abstract}
Matrix-free polymer grafted nanoparticles (NPs) are single component polymer nanocomposites (PNCs) for which the often reported severe aggregation of the conventional PNCs can be suppressed. For a given particle core, the size and shape of the polymer grafted nanoparticles can be controlled by the molecular weight of the polymer and its grafting density. However, the degree of homogeneity of one-component PNCs depends on the grafted chain molecular weight and grafting density, as well as on the shape of grafted NPs. Surface plasmon resonance enhanced dynamic light scattering from very dilute solutions, yielding both translational and rotational transport coefficients, complemented by UV-Vis extinction spectra, can detect deviations from spheres. Here, we report that poly(isobutylene)grafted Ag NPs strongly deviate from the spherical shape and are modeled as prolate spheroids. This NP asphericity, due to inhomogeneous grafting, can impact the structure and properties of plasmonic PNCs in the solid state. Thus, characterizing this behavior is a crucial step prior to the formation of one-component PNCs.
\end{abstract}

Published under license by AIP Publishing. https://doi.org/10.1063/1.5140459

\section{INTRODUCTION}

Matrix-free (one-component) polymer nanocomposites (PNCs) are of substantial interest in fundamental research and industrial applications, as they can combine the highly effective material properties of the two components at the local confinement. ${ }^{1,2}$ The hardsoft combination of the composites can lead to improved mechanical, electrical, and optical properties of materials. ${ }^{3-9}$ Within the class of PNCs, ${ }^{10-17}$ polymer shell/rigid core metal is of uprising interest as the polymer corona can provide uniformity, stability, and better dispersibility, and can even solubilize metallic nanoparticles (NPs). ${ }^{17}$ Engineering PNCs for optical applications, as an example, requires high optical transparency at high filling ratios of nanoparticles. ${ }^{16}$ By utilizing transparent polymers at a high grafting density to the metal core, even optically clear PNCs have been reported. ${ }^{16}$ On the other hand, sparse particle brushes have led to enhanced physical properties of solid composite materials, with pronounced polymer-polymer interactions of the grafted coil-like chains. ${ }^{6,9}$
Combining transparent and elastomeric polymers tethered onto metallic cores at a low grafting density could, thus, lead to interesting physical properties at both solid and solution states.

Well-defined materials prepared by living cationic polymerization ${ }^{18-20}$ offer exceptional abilities in the preparation of model polymers with control of structure tuning and can be used as efficient dispersants of metallic NPs. ${ }^{17,21,22}$ Synthetic functional poly(isobutylenes) (SFPIBs) are chain-end functionalized transparent materials that can act as soft elastomeric templates tethering hard spheres (e.g., PIB-SH) for addressing novel physical properties related to the behavior of grafted plasmonic NPs in solution, ${ }^{5,23,24}$ and their laser light-stimulated photoacoustic response in the solid state. $^{6-9,25}$ To explore a gap in the literature, where mainly the interaction of light with grafted glassy polymers has been tested, ${ }^{4-9}$ or that of rubbery polymers attached onto silica NPs, ${ }^{26}$ the structure/property interplay and optomechanical properties of rubbery, PIB-tethered metallic particles should be investigated. 
Plasmonic NPs such as Au and Ag are polycrystalline facets due to synthetic routes deviating from the spherical shape, as typically adopted by polymeric colloids, and possess large size polydispersity. Hence, characterization prior to the formation of onecomponent PNCs is crucial. Low polymer grafting density and long grafts have been shown computationally and experimentally to promote NP anisotropy at a constant core size. ${ }^{5,23,24,27}$ While bare $\mathrm{Au}$ cores assume an almost spherical shape, asphericity and surface inhomogeneity occur upon their soft grafting. ${ }^{5}$ In uniformly coated $\mathrm{CH}_{3}-\left(\mathrm{CH}_{2}\right)_{\mathrm{x}} \mathrm{S}-\mathrm{Au}$ nanospheres, the free volume that the grafted polymeric chains have to explore (due to NP curvature) has been suggested to dictate the coating's spherical symmetry and stability. ${ }^{23}$ For Au NP core sizes of $8 \mathrm{~nm}$, simulations have shown moderate asymmetric coating arrangements in solution, unlike smaller cores $(2 \mathrm{~nm})$, where the asymmetry and surface coating inhomogeneity (creating patches) is higher, and thus aggregation is difficult to prevent. ${ }^{23,24}$ As NP aggregation has to be avoided for many applications, e.g., optically transparent materials, ${ }^{16,22}$ the grafting arrangement and shape asphericity of NPs with metal cores larger than $10 \mathrm{~nm}$ is crucial to determine.

Herein, we investigate the shape of brush NPs consisting of spherical Ag cores $(\sim 20 \mathrm{~nm})$ stabilized by thiol-terminated PIB chains of a low molecular weight $(2.500 \mathrm{~g} / \mathrm{mol})$ at two different grafting densities $(20 \%$ and $65 \% \mathrm{Ag})$ in very dilute solutions, by dynamic polarized and depolarized light scattering and UV/Vis absorption. Dynamic light scattering (DLS) complemented by UV-Vis extinction spectra is the method of choice that requires very low concentrations $(\sim 0.02 \mathrm{~g} / \mathrm{l})$ to reduce the inherent light absorption, ${ }^{5}$ while the presence of plasmonic resonance compensates the reduced signal. The resonance enhancement is strong if the wavelength of the laser used in the DLS experiment commensurates the wavelength at the maximum extinction of the UV-Vis spectrum. ${ }^{5,28}$ PIB was chosen because of its low glass transition temperature $\left(\mathrm{T}_{\mathrm{g}}\right)$, which allows for the exploration of the viscoelastic behavior of matrix-free PNCs at ambient conditions. Emphasis is given to the transparency and elasticity of the PIB ligand, its narrow molecular weight distribution (importance of efficient synthetic capabilities and model polymers), as well as to the extensive removal of unbound polymeric chains for the determination of the dynamic and physical properties of synthetic PIB brush-grafted particles. Plasmonic NPs with inherent less spherical and regular size and shape compared to PS or silica colloids were found, extensively characterized, and analyzed in dilute solution.

\section{EXPERIMENTAL DETAILS}

\section{A. Preparation of PIB-capped Ag-nanoparticles}

Olefin-terminated PIB was initially synthesized and converted to PIB-SH (see the supplementary material), which was used as a stabilizer for grafting Ag NPs after ligand exchange. In a typical procedure, $1 \mathrm{ml}$ of a $5 \mathrm{wt}$. \% water dispersed poly(vinylpyrrolidone) (PVP)-capped Ag NPs (stock dispersion; purchased by US Research Nanomaterials, Inc.) was diluted with $9 \mathrm{ml}$ methanol (leading to $50 \mathrm{mg}$ of PVP-Ag overall). The mixture was slowly added to a $40 \mathrm{ml}$ toluene solution of PIB-SH $(400 \mathrm{mg}, 0.154 \mathrm{mmol}$, $2600 \mathrm{~g} / \mathrm{mol}$ ) at room temperature. Methanol was left to evaporate in the hood, and the system was left to react for 3 days under stirring, at the same conditions, transferring the Ag NPs into the PIB matrix. The toluene was then removed by rotor evaporation; the crude mixture was dispersed in a small amount of DCM and precipitated in methanol to remove PVP. The mixture was then dissolved in $200 \mathrm{ml}$ hexane and extracted with methanol. The hexane phase containing the yellow PIB-Ag solution was collected, dried with sodium sulfate, filtrated, and rotovapped. The mixture was finally dispersed in $30 \mathrm{ml}$ hexane and centrifuged at $12000 \mathrm{rpm}$ for $2 \mathrm{~h}$ to remove unbound PIB-SH (supernatant) and concentrate the PIB-Ag (nanocomposite precipitate). The procedure was repeated one more time (two centrifugation rounds overall). The final residue was then dried in a vacuum oven at $60^{\circ} \mathrm{C}$ for $12 \mathrm{~h}$.

\section{B. Dynamic light scattering (DLS)}

The normalized light scattering intensity $[I(q, t)]$ autocorrelation function, $G(q, t) \equiv\langle I(q, t) I(q)\rangle /|I(q)|^{2}$, was recorded over a broad time range $\left(\mathrm{t}=10^{-7}-10^{3} \mathrm{~s}\right)$ at different scattering wave vectors $q$ with an ALV/LSE-5004 goniometer/correlator setup using an He-Ne laser with wavelength $\lambda=632 \mathrm{~nm}$. The scattering vector $\boldsymbol{q}=\boldsymbol{k}_{\boldsymbol{s}}-\boldsymbol{k}_{\boldsymbol{i}}$ with $\boldsymbol{k}_{\boldsymbol{s}}$ and $\boldsymbol{k}_{\boldsymbol{i}}$ being the wave vectors of the scattered light and the incident laser light, respectively, has magnitude $q=(4 \pi n / \lambda) \sin (\theta / 2)$ ( $n$ and $\theta$ are the solution refractive index and the scattering angle, respectively). For ergodic systems, the scattered field correlation, $C(q, t)=\left\{[G(q, t)-1] / f^{*}\right\}^{1 / 2}$, is computed from the intensity correlation and $f^{\star} \leq 1$ is a coherence instrumental factor. We have performed polarized (VV) and depolarized (VH) DLS experiments using a vertically $(\mathrm{V})$ polarized incident laser beam and selected the scattered light polarized vertically (VV-configuration) and horizontally (VH-configuration) to the scattering plane $\left(k_{i}, k_{f}\right)$. For spherical monodisperse nanoparticles with zero optical anisotropy, no $\mathrm{VH}$ scattering can be observed, and the translational diffusion coefficient $D_{t}=\Gamma(q) / q^{2}$ is directly obtained from the exponential decay rate, $\Gamma(q)$, of the experimental $C_{V V}(q, t)$. For anisotropic particles with finite optical anisotropy, the experimental $C_{V H}(q, t)$ is a single exponential decay, whereas the corresponding $C_{V V}(q, t)$ becomes bimodal. In principle, $C_{V V}(q, t)$ includes both isotropic and anisotropic scattering contributions, but it is usually unimodal because the anisotropic scattering is much weaker. However, the strong surface plasmon enhanced depolarized light scattering renders the anisotropic contribution to $C_{V V}(q, t)$ discernible. In this case, the rotational, $D_{\mathrm{r}}$ and translational, $D_{t}$ diffusion coefficients can be obtained from the experimental $C_{V H}(q, t)$ and $C_{V V}(q, t)$ relaxation functions. Measurements were carried out at $20^{\circ} \mathrm{C}$.

\section{RESULTS AND DISCUSSION}

Olefin-terminated PIB (denoted as $\mathrm{PIB}_{2.5 \mathrm{~K}}$-olefin) was initially synthesized in large scale $(\sim 6 \mathrm{~g})$ via living cationic polymerization $\left(M_{n}=2.500 \mathrm{~g} / \mathrm{mol}, \mathrm{Ð}=1.38\right.$; five columns SEC: size-exclusion chromatography; $Đ=1.14$ at three columns SEC). Photoinitiated thiol-ene coupling was then used to modify the end-chain olefin to thiol, as shown in Scheme 1. After postmodification of PIB-olefin to PIB-SH, there was a negligible change in the molecular weight of the polymer $(2600 \mathrm{~g} / \mathrm{mol})$, while the dispersity $(Đ)$ slightly changed from 1.38 to 1.39 (Fig. S1 in the supplementary material). The molecular structures of the polymers before and after modification were confirmed by ${ }^{1} \mathrm{H}$ NMR spectroscopy (Fig. S2 in the 
PIB-olefin synthesis

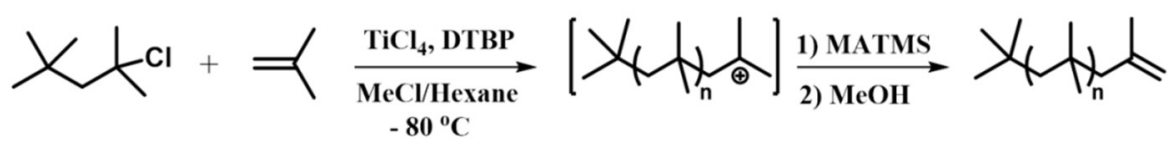

PIB-SH synthesis<smiles>C=C(C)CC(C)CC(C)(C)CC(C)(C)C</smiles><smiles>CC(C)C(C)C(C)C(C)CSCCCS</smiles>

SCHEME 1. Synthetic scheme for the preparation of olefin-terminated PIB and post-modification with 1,3-propanedithiol to yield thiol-terminated PIB, which was used as a stabilizer for Ag NPs.

PIB-stabilized AgNPs

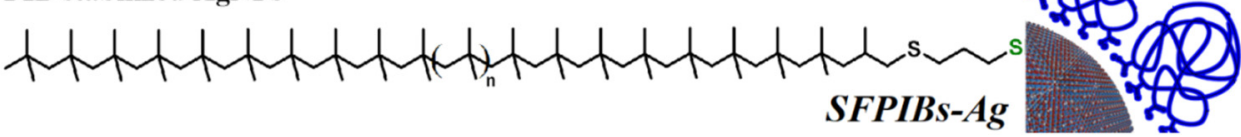

supplementary material). PIB-SH was then used to cap premade PVP-stabilized Ag NPs, taking advantage of the strong binding of the thiol group to the NP surface, which acted as the driving force for ligand exchange with the physically coordinated (tertiary amide groups) PVP shell. ${ }^{29}$

To prepare PIB-S-Ag NP hybrid materials, PVP-stabilized Ag NPs were ligand exchanged to PIB-SH. By varying the PVP-Ag NPs/PIB-SH ratio, two different percentages of $\mathrm{Ag}(20 \%$ and $65 \%)$ were obtained (analytical characterization in the supplementary material). Following precipitation and extraction with methanol to remove fully the liberated PVP (soluble in methanol), an additional purification step was performed, as described in Sec. II. Impurity-free, PIB-stabilized Ag NPs were additionally centrifuged in hexane at a high speed (twice) to remove unbound PIB chains (centrifugal fractionation). This step seemed to be crucial for material purification and physical properties. A similar procedure has been reported in the literature. ${ }^{30,31}$ Thermogravimetric analysis (TGA) results of the starting material, PVP-Ag, showed a $72 \mathrm{wt} \%$ grafting of PVP to the Ag core (Fig. S3 in the supplementary material), while on the other hand, the two PIB-Ag synthetic formulations denoted as $\mathrm{PIB}-\mathrm{Ag}_{20 \%}$ and $\mathrm{PIB}-\mathrm{Ag}_{65 \%}$, showed a metal content of 20 wt. \% and 65 wt. \% respectively (Fig. S4 and Table S1 in the supplementary material). Analysis of the TGA thermographs also indicated the attachment of PIB onto NPs; the degradation temperature $\left(T_{d}\right)$ of PIB-Ag increased in the composite from 405 to $417^{\circ} \mathrm{C}$ as observed from Fig. S4 in the supplementary material, while unbound PIB chains were found in the supernatant at every centrifugation round [Fig. 1(a)]. The grafting density $(\sigma)$ of the PIB- $\mathrm{Ag}_{20 \%}$ and PIB- $\mathrm{Ag}_{65 \%}$ samples can be also expressed as the number of PIB chains $/ \mathrm{nm}^{2}$, assuming homogeneous grafting, $\sigma=\left(\right.$ wt. $\left.\%_{\text {PIB }} /\left(1-w t . \%_{\text {PIB }}\right)\left(\rho^{\star} \mathrm{N}_{\mathrm{A}}\right) / \mathrm{MW}_{\mathrm{PIB}}\right)(\mathrm{V} / \mathrm{S})$, where $\quad \mathrm{N}_{\mathrm{A}}$ is Avogadro's number, $\rho$ is the Ag density, and S/V is the surface to volume ratio of the Ag NPs. Given the deviation of the Ag NPs from the spherical shape (see below) and the high grafting density, the absolute $\sigma$ values are subject to errors. ${ }^{32}$ Much more reliable is the relative grafting density among the two systems, with the PIB- $\mathrm{Ag}_{20 \%}$ sample to be about seven times densely grafted than the PIB- $\mathrm{Ag}_{65 \%}$.

The higher percentage of Ag NPs (65\%) in the PIB composite was also confirmed by UV-Vis spectroscopy [Fig. 1(b)]. The
PIB-Ag ${ }_{65 \%}$ exhibited almost six times higher absorbance than PVP-Ag in $\mathrm{CHCl}_{3}$, when measured at the same concentration $(0.05 \mathrm{mg} / \mathrm{ml})$. The maximum absorbance wavelength remained the same at $433 \mathrm{~nm}$; however, the full width at the half maxima
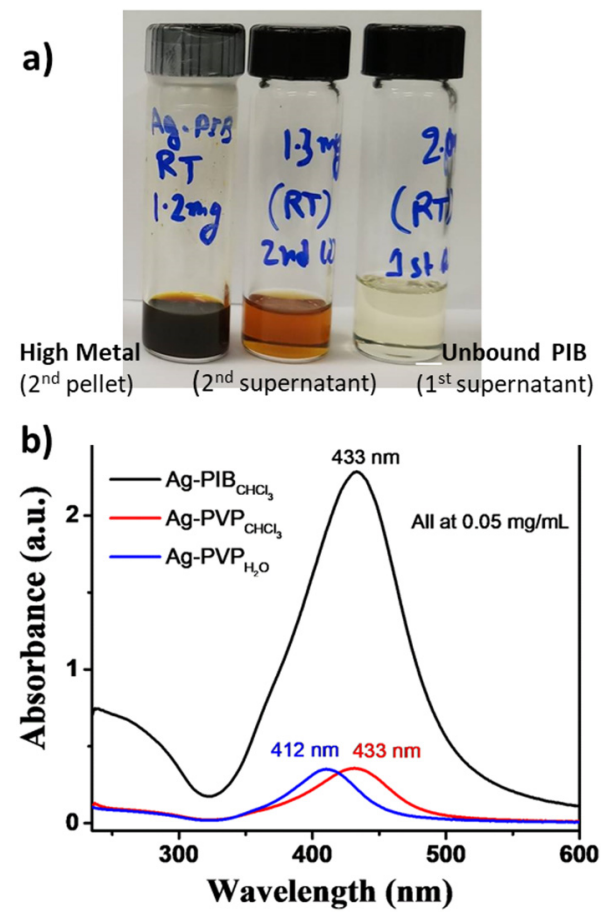

FIG. 1. a) Photos of PIB-S- $\mathrm{Ag}_{65}$ after two centrifugation rounds in hexane; high metal PIB-Ag ( $2^{\text {nd }}$ nanocomposite precipitate) along with the $1^{\text {st }}$ and $2^{\text {nd }}$ supernatant solutions containing unbound PIB. (b) Experimental UV-Vis spectra of PIB-S- $\mathrm{Ag}_{65} \% 2^{\text {nd }}$ precipitate (black line) and PVP-Ag precursor (red line) in chloroform at $0.05 \mathrm{mg} / \mathrm{ml}$. The spectrum of PVP-Ag in water is also shown at the same concentration for reference. A similar UV-Vis spectrum as PVP-Ag was obtained for the $1^{\text {st }} \mathrm{PIB}-\mathrm{Ag}$ nanocomposite precipitate (not shown for clarity). Supernatant PIB solutions did not have any absorbance. 


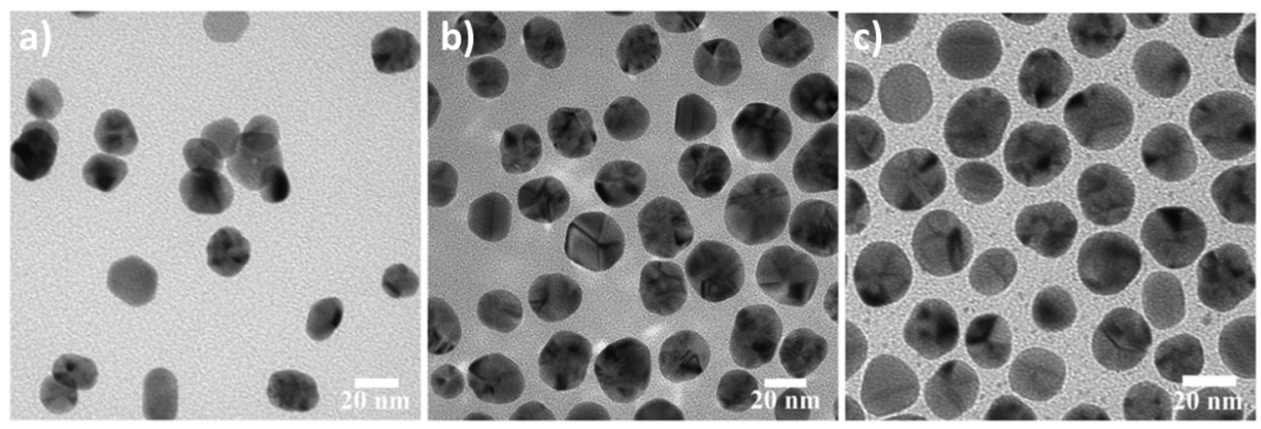

FIG. 2. TEM image of (a) PVP-Ag, (b) PIB-S-Ag $20 \%$, and (c) PIB-S-Ag $\mathrm{Ag}_{65}$. changed from $70 \mathrm{~nm}$ to $88 \mathrm{~nm}$ as the PVP-stabilizer was exchanged to PIB in Ag NPs. The importance of the extra purification step by centrifugation is shown in Fig. 1(a). The $1^{\text {st }}$ supernatant hexane fraction, post ligand exchange, precipitation, and extraction, contained a significant amount of free PIB chains. Purifying the system with a second centrifugation round led to a nanocomposite with a high metal content (denoted as $2^{\text {nd }}$ nanocomposite precipitate), as well as with a $2^{\text {nd }}$ supernatant with a lower fraction of unbound PIB compared to the $1^{\text {st }}$ one. Both supernatant solutions [Fig. 1(a)] were characterized by UV-Vis [Fig. 1(b)] and TGA (Fig. S4 and Table $S 1$ in the supplementary material), and finally decanted, and the high metal, PIB-stabilized Ag NP fractions were isolated and utilized for our solution studies. Transmission electron microscopy results (TEM; experimental details in the supplementary material) showed a minimal change in size, from $21.2 \pm 3.1 \mathrm{~nm}$ in PVP-Ag to $22.5 \pm 3.3 \mathrm{~nm}$ and $21.6 \pm 3.3 \mathrm{~nm}$ in the case of PIB-S- $\mathrm{Ag}_{20 \%}$ and PIB-S-Ag ${ }_{65 \%}$, respectively (Fig. 2), denoting successful ligand exchange. The uniform distribution and well-defined interparticle distance in the case of PIB-stabilized Ag NPs, relative to PVP-Ag, is attributed to the removal of unbound polymer chains, as shown in Fig. 2, since the sample preparation conditions were the same.

For $\mathrm{Ag}$ nanoprisms ${ }^{33}$ and $\mathrm{Au}$ nanorods, ${ }^{34}$ dynamic depolarized light scattering is significantly enhanced near the localized surface plasmon resonance (LSPR) frequency. Anisotropic dynamic light scattering was also reported for Au nanospheres, ${ }^{5}$ due to their inherent crystallinity. ${ }^{34}$ Based on the TEM mages, the present Ag core NPs display both size and shape polydispersity (Fig. 2). Polymer tethering can also influence their shape due to surface asymmetry effects induced by grafting (even for spherical NPs) and can lead to deviations from the spherical symmetry producing asymmetric configurations. ${ }^{23,35,36}$ Experimental support of the anisotropic shape of otherwise isotropically grafted Au NPs has been reported, utilizing LSPR enhanced dynamic polarized and depolarized light scattering and UV-Vis extinction spectra. ${ }^{5}$ Such spatially anisotropic graft distribution can impact the assembly of these apparently spherical NPs in undiluted solid films. ${ }^{27,37,38}$

The two PIB-grafted Ag (Ag@PIB) NPs display one broad resonance with a maximum corresponding to the excitation of the LSPR mode inside the Ag core [Fig. 1(b)]. Due to polydispersity in size and shape, this curve results from the superposition of extinction spectra of slightly spheroidal Ag NPs, which exhibit mostly two modes along each of the short and long axes. The maximum in Fig. 1(b) corresponds to the most intense long-axis plasmon, whereas a small shoulder can be noticed close to $400 \mathrm{~nm}$, which is the signature of the short axis plasmon. This information will be used below to numerically estimate the average shape of the Ag core. The latter can be sensitively addressed by DLS, both polarized and depolarized, probing the transport coefficients of the grafted $\mathrm{Ag}$ NPs at very low concentrations $(0.02 \mathrm{~g} / \mathrm{l})$. Even at these concentrations, the depolarized scattering intensity at $632 \mathrm{~nm}$ is high enough ( $\sim 7 \%$ of the polarized intensity, Fig. S5 in the supplementary material) to record the orientation relaxation function $C_{V H}(q, t)$. The latter is shown in comparison with the corresponding $C_{V V}(q, t)$ at two different q's and $20^{\circ} \mathrm{C}$ in Fig. 3(a). The orientation function, $C_{V H}(q, t)=\alpha \exp \left[-\Gamma_{f} t\right]$, exhibits a single exponential decay [black solid lines in Fig. 3(a)] with relaxation rate $\Gamma_{f}(q)=6 D_{r}+q^{2} D_{t}$ [solid lines in the lower panel of Fig. 3(b)] and hence $D_{r}=\Gamma_{f}(\mathrm{q} \rightarrow 0) / 6$ is directly obtained from the intercept of $\Gamma_{f}(\mathrm{q} \rightarrow 0)$. For plasmonic metals, ${ }^{5,33,34}$ the contribution of the LSPR enhanced depolarized scattering in $C_{V V}(q, t)=a_{f} \exp \left[-\Gamma_{f} t\right]+a_{s} \exp$ $\left[-\Gamma_{s} t\right]$ appears as a second fast decay [Fig. 3(a)] with amplitude $\alpha_{\mathrm{f}}$ and relaxation rate $\Gamma_{\mathrm{f}}$. The slow purely isotropic component with amplitude $\alpha_{s}$ decays via translational diffusion, allowing for a precise determination of $D_{t}$ from the slope of the linear dependence, $\Gamma_{s}(\mathrm{q})=\mathrm{q}^{2} \mathrm{D}_{\mathrm{t}}$ [upper panel of Fig. 3(b)]. From $D_{t}$ and $D_{r}$, we compute the hydrodynamic radius $R_{h}=k_{B} T /\left(6 \pi \eta_{s} D_{t}\right)$ and the rotational radius $R_{r}=\left[\mathrm{k}_{\mathrm{B}} \mathrm{T} /\left(8 \pi \eta_{s} D_{r}\right]^{1 / 3}\right.$ for a sphere under stick boundary conditions, where $k_{B}$ is Boltzmann's constant and $\eta_{s}$ is the solvent viscosity. In the opposite case of slip boundary conditions, $R_{h}$ and, in particular, $R_{r}$ would assume unacceptable much higher values in order to conform to the two experimental transport coefficients. ${ }^{39,40}$ We also note that, for non-spherical NPs, depolarized DLS selectively probes the reorientation of the long axis as schematically shown for Ag@PIB NP with a prolate ellipsoidal shape in Fig. 3(c).

The exponential shape of the two relaxation functions seems to be rather unexpected in view of the significant size polydispersity (Fig. 2) of the Ag core being the same in both systems. In the DLS experiment, the laser wavelength $(632 \mathrm{~nm})$ exceeds the wavelength $\lambda_{\max }(\sim 435 \mathrm{~nm})$ of the LSPR peak in the extinction spectrum of the Ag@PIB NPs in toluene [Fig. 4(a)]. The relaxation functions of Fig. 3 recorded at a longer photon wavelength than $\lambda_{\max }$ represent a narrow fraction of the Ag@PIB NP distribution, selected by the LSPR enhanced scattering at $632 \mathrm{~nm}$ resulting in their exponential decay. In particular, $\mathrm{C}_{\mathrm{VH}}(\mathrm{q}, \mathrm{t})$ is more sensitive to the size distribution as $\Gamma_{f} \sim R_{r}{ }^{-3}$ compared to $\Gamma_{s} \sim R_{h}{ }^{-1}$ and its exponential shape 

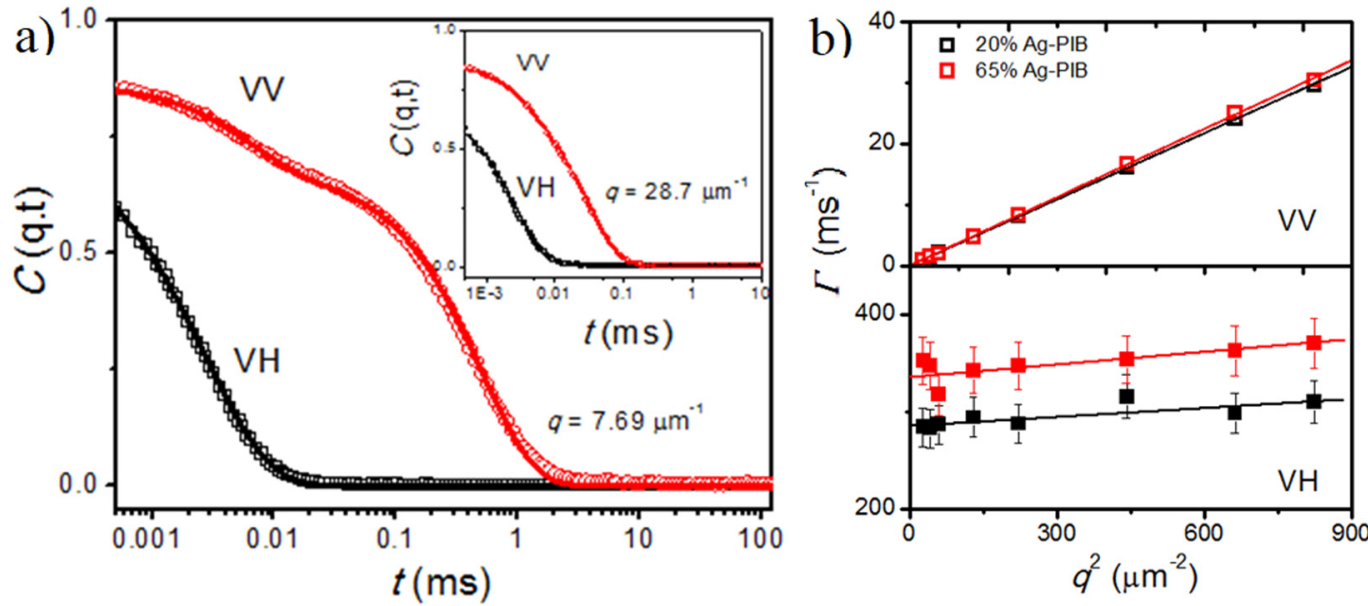

c)

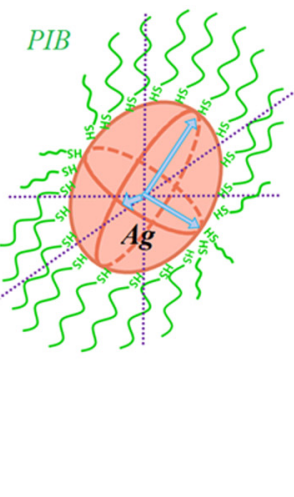

FIG. 3. (a) Experimental polarized (VV, red circles) and depolarized (VH, black squares) relaxation functions (at two different scattering wave vectors, q, for Ag@PIB $(65 \%$ $\mathrm{Ag} @ \mathrm{PIB}, \mathrm{c}=0.02 \mathrm{~g} / \mathrm{l})$ in toluene at $20^{\circ} \mathrm{C}$. The depolarized $\mathrm{C}_{\mathrm{VH}}(\mathrm{q}, \mathrm{t})$ function can be represented by a single exponential decay (black lines), whereas two relaxation processes (red solid lines) contribute to the polarized $C_{V v}(q, t)$ function. Inset: the proximity of the $C_{v v}(q, t)$ decreases with increasing $q$ due to the speed-up of the slow process. (b) The relaxation rate $\Gamma_{s}(q)$ (upper panel) and $\Gamma_{f}(q)$ obtained from $\mathrm{C}_{\mathrm{VV}}(\mathrm{q}, \mathrm{t})$ (lower panel) and $\mathrm{C}_{\mathrm{VH}}(\mathrm{q}, \mathrm{t})$, respectively. (c) Ag@PIB NPs are represented by a prolate ellipsoid for which only the reorientation of the long axis is depolarized light scattering active.

[Fig. 3(a), black symbols and solid line] is a strong indication of biased wavelength selection. In addition, the grafting of the Ag@PIB NPs should enhance interactions between surface and the surrounding solvent, and stick boundary conditions are, therefore, more appropriate. ${ }^{5}$ For the high polymer grafting density Ag@PIB NPs (20 wt. $\%$ Ag or 2.3 vol. \%), $R_{h}=10.0 \pm 0.2 \mathrm{~nm}$ and $R_{r}=17.9 \pm 0.4 \mathrm{~nm}$. In comparison, $R_{h}=9.7 \pm 0.2 \mathrm{~nm}$ and $R_{r}=16.9 \pm 0.3 \mathrm{~nm}$ are computed for the lower polymer grafting density Ag@PIB NPs (65 wt. \% Ag or $15 \mathrm{vol} . \%$ ). The disparity is distinct for the $R_{\mathrm{r}}$ radii due to the stronger size dependence of $D_{r}\left(\sim R_{r}^{-3}\right)$ than $D_{t}\left(\sim R_{h}{ }^{-1}\right)$. These values should contrast with the average radius of the $\mathrm{Ag}$ $\mathrm{NP}$ core obtained from TEM: $R_{c}=11.3 \pm 1.7 \mathrm{~nm}$ (for $20 \% \mathrm{Ag}$ );
$R_{c}=10.8 \pm 1.7 \mathrm{~nm}$ (for $65 \% \mathrm{Ag}$ ). Albeit within error, the higher grafting seems to occur on slightly larger Ag cores as also suggested by the wavelengths at the maximum extinction [see Fig. 4(a) below]. Since both Ag@PIB NPs have similar Ag cores and PIB degree of polymerization $\mathrm{N}=46$, the shell thickness should be larger for the 20\% Ag@PIB NPs with a higher grafting density than 65\% Ag@PIB as reflected in their $R_{r}$ values.

The unexpected finding, $R_{h}<R_{r}$ for both examined Ag@PIB NPs is emphasized recalling that $R_{h}<R_{r}$ is not compatible with the spherical symmetry of grafted NPs. Instead, implementation of anisotropic graft shape is also found to account for the difference between the two radii. For an adequate description of $D_{r}$ by Perrin's
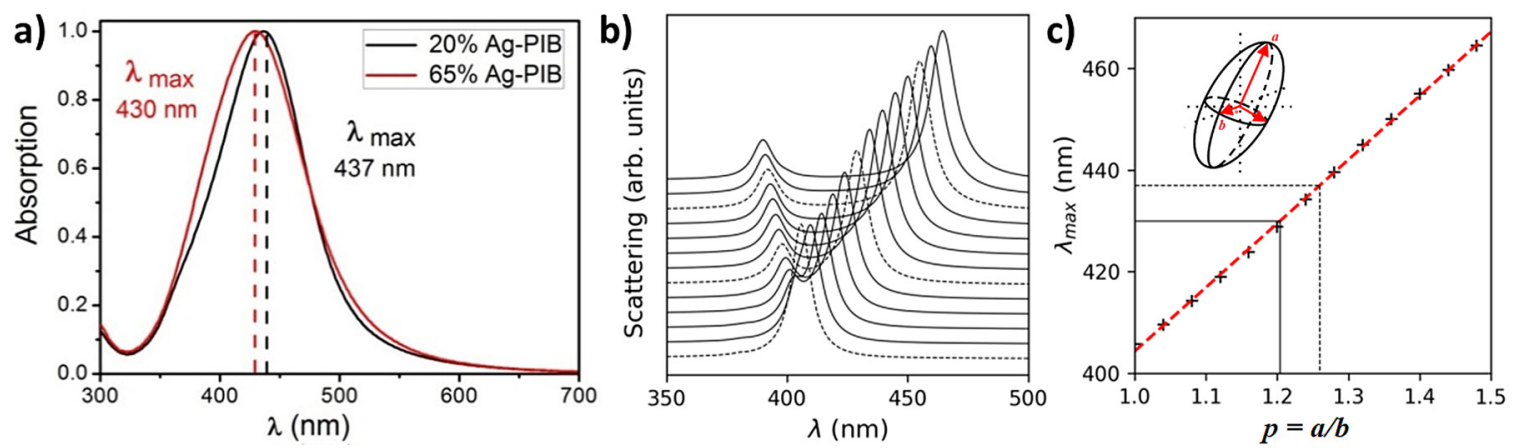

FIG. 4. (a) Experimental UV-Vis spectra (black and red curves) of the two Ag@PIB samples in toluene (0.02 g/l). (b) Computed extinction spectra of a single Ag core nanoparticle in toluene for different aspect ratios, $p$, ranging between 1.04 and 1.48 with step 0.04 , from bottom to top. (c) Evolution of the plasmon resonance wavelength [in (b)] associated with the long-axis surface plasmon mode with $p$. The red dashed line is a linear fit. Solid and dashed black lines indicate the correspondence between the experimental plasmon resonance wavelengths 430 and $437 \mathrm{~nm}$ in (a) with $p=1.20$ and 1.26 , respectively. Inset: a schematic of a prolate ellipsoid (Ag core) with a and $b$ being the length of the long and short axes, respectively. Due to the very low optical contrast, PIB has negligible contribution to the extinction spectrum. 
equation for a prolate ellipsoid (supplementary material S6) ${ }^{41}$ [Fig. 3(c)], $R_{h}=\mathrm{b} / \mathrm{S}$ and $R_{r}=(\kappa g \perp \mathrm{p})^{1 / 3} b$, where $p=a / b$ is the aspect ratio with $a$ and $b$ being the length of the long and short axes, respectively, and the parameters $\mathrm{S}, \mathrm{g} \perp$ are functions of $p$. The hydrodynamic volume, $V_{h}=\kappa V_{o}$ where $V_{0}=(4 / 3) \pi a b^{2}$ is the geometrical volume and $\kappa$ is a dimensionless parameter depending on the ratio $R_{r} / R_{h}$. Hence, this ratio, $1<\left(\mathrm{R}_{\mathrm{r}} / \mathrm{R}_{\mathrm{h}}\right)=\mathrm{S}(\kappa \mathrm{g} \perp \mathrm{p})^{1 / 3} \approx \kappa^{1 / 3}$ implies $\kappa \geq 1$ noting that for $p=1$ (sphere), $\mathrm{S}$ and $\mathrm{g} \perp$ are equal to 1 and, therefore, $R_{r}=R_{h}$. Since there are three unknown parameters $(a, b$, and $\kappa)$, we follow the procedure in Ref. 5, assuming, $p=1.2, \mathrm{~S}=0.93$, and $\mathrm{g}_{\perp}=1.09$. Hence, $b=9.3 \mathrm{~nm}$ and $a=11.2 \mathrm{~nm}$, and the ratio $R_{r} / R_{h}$ assume numerical values of $1.79(\kappa=5.5)$ and $1.74(\kappa=5.1)$ for the higher (20\%) and lower (65\%) PIB-grafted Ag NPs, respectively; the value of $\kappa$ slightly decreases with increasing $p$. For comparison, lower ratio $\mathrm{R}_{\mathrm{r}} / \mathrm{R}_{\mathrm{h}}=1.15-1.35$ values and hence $\kappa(=1.5-2.4)$ have been reported for grafted $\mathrm{Au} \mathrm{NPs}{ }^{5}$ We should notice that the direct access to both transport coefficients $\left(D_{t}, D_{r}\right)$ and hence to $R_{h}$ and $R_{r}$ is best provided by the surface plasmon resonance enhanced polarized and depolarized light scattering. Fluorescence correlation spectroscopy is an alternative experimental technique for the measurement of $D_{t}$ and under conditions (nanorods) of $D_{r}$ based, however, on a single length scale (focus spot). ${ }^{42,43}$ Owing to this limitation, the diffusion nature should be assumed. ${ }^{44}$

The much higher $\kappa$ value in the present PIB-grafted Ag NPs is remarkable and in the framework of an orientation of a prolate ellipsoid [Fig. 3(c)] normal to the long axis, this finding could imply a irregular Ag core shape with strong hydrodynamic friction. The shape anisotropy of PIB-tethered Ag NPs could be due to the high PIB solubility in organic solvents (even in hexane), which may solubilize the external surface of the Ag core to some extent (preferentially to one axis), conforming to a prolate spheroid [Fig. 3(c)]. The experimental $R_{h}$ values $(\approx 10 \mathrm{~nm})$ are very similar to the core radius, $R_{A g \text {-core }} \approx 11 \mathrm{~nm}$, obtained by TEM. The PIB layer thickness approximated by the end-to-end distance $R_{e}$ of the utilized PIB-SH $\left(\mathrm{N}_{\mathrm{PIB}}=46\right)$ can be estimated for two chain conformations; Gaussian at low and stretched at low and high grafting densities, respectively. $R_{e}=\mathrm{bN}^{0.5} \approx 3 \mathrm{~nm}$ for Gaussian, and $R_{e}=\mathrm{bN}^{0.7}$ $\approx 7 \mathrm{~nm}$ for stretched conformation, are estimated using a monomer length, $\mathrm{b}=0.5 \mathrm{~nm}$. Hence, the total size should range between $14 \mathrm{~nm}$ and $18 \mathrm{~nm}\left(R_{\text {Ag-core }} \approx 11 \mathrm{~nm}\right)$. While these values are comparable to the rotational radius, $R_{r}$, the hydrodynamic $R_{h}$ assumes a much lower value than its rough estimate, probably due to the significant NP asphericity.

In addition to TEM (Fig. 2), information about the Ag core shape could be obtained from the extinction spectra of PIB-stabilized Ag NPs in toluene, as shown in Fig. 4(a), where the maximum is reached for a wavelength $\lambda_{\text {max }}$ close to $430 \mathrm{~nm}$, related to surface plasmon excitation in the Ag core. The extinction values $\lambda_{\text {max }}$ in Fig. 4(a) were simulated assuming Ag core asphericity [Fig. 4(b)]. We employed the finite element method (Comsol Multiphysics) to evaluate numerically the effect of Ag core asphericity on the surface plasmon resonance. Due to the very small difference in the refractive index between PIB and toluene, the grafting induces a wavelength shift smaller than $1 \mathrm{~nm}$ and was not included in the model. The variation of the aspect ratio ( $p$ ) of a prolate $\mathrm{Ag}$ nanoparticle between 1 and 1.5 (with short axis $\mathrm{b}=10 \mathrm{~nm}$ ) induces a wavelength redshift of the long-axis plasmon mode from 405 to $465 \mathrm{~nm}$ [see Fig. 4(b); the shifted peak corresponds to the short axis surface plasmon]. As shown in Fig. 4(c), the redshift is linear with a slope of $125.6 \mathrm{~nm} / p$. The experimental LSPR resonance wavelength, $\lambda_{\max }$, can be captured for a prolate Ag shape with $b=10 \mathrm{~nm}$ and $a=12.0 \mathrm{~nm}$ for Ag@PIB NPs (65 wt.\% Ag, $\lambda_{\text {max }}=430 \mathrm{~nm}$ and $p=1.20$ ), and $a=12.6 \mathrm{~nm}$ for the higher grafting density Ag@PIB NPs (20wt. \% Ag, $\lambda_{\max }=437 \mathrm{~nm}$ and $p=1.26)$. It seems that the $\mathrm{Ag}$ core is, in average, slightly more elongated in solution for NPs with a higher polymer grafting density. A possible reason is that those NPs have slightly higher probability to bind with a large number of PIB-SH chains and are preferentially selected in the preparation process. In other words, the observed wavelength shift could be a consequence of the fact that PIB-SH has different reactivities to Ag core facets, with $20 \%$ Ag grafting leading to the selection of more irregular shapes. Moreover, the relatively large ratio $\mathrm{R}_{\mathrm{r}} / \mathrm{R}_{\mathrm{h}}$ suggests that the Ag@PIB NPs should possess somewhat a higher asphericity than their cores probably due to the anisotropic grafting of $\mathrm{Ag} \mathrm{NP}$ along the two main axes as schematically shown in Fig. 3(c).

\section{CONCLUDING REMARKS}

The interaction of light with matrix-free PNCs in dilute solution was herein explored, combining the transparency and rubbery behavior of SFPIBs, prepared by living cationic polymerization, with the localized surface plasmon resonance behavior of Ag NPs (hard spheres). To define the anisotropies derived from NPs polycrystallinity and polymer soft grafting in one-component PNCs, a detailed size and shape characterization was performed. We utilized both polarized and depolarized DLS, which due to the surface plasmon enhancement, even about $100 \mathrm{~nm}$ off-resonance, gains high sensitivity. It enables the measurement of NPs rotational diffusion at very dilute concentrations $(\sim 0.02 \mathrm{~g} / \mathrm{l})$ in the absence of $\mathrm{NP}$ aggregation. PIB-olefin of $2,500 \mathrm{~g} / \mathrm{mol}$ was synthesized in large scale $(\sim 6 \mathrm{~g})$ and converted to PIB-SH, which was used as the stabilizer for the two Ag@PIB NPs (20\% Ag and 65\% Ag). An unexpected high rotation radius $R_{r}>R_{h}$ was found for both examined PIB-stabilized Ag NPs, with the same PIB degree of polymerization $(\mathrm{N}=46)$, but different grafting densities (20\% Ag and 65\% Ag), with small size differences between the two samples. Rather unexpected was the significant smaller value of the hydrodynamic radius, $R_{h}$ (approaching $R_{\text {core }}$ values) that indicated strong deviation from the spherical shape. In fact, the experimentally obtained large ratio $R_{r} / R_{h} \sim 1.76$ can be rationalized by a prolate ellipsoid, with hydrodynamic volume that significantly exceeds the geometrical volume of Ag@PIB NPs. Due to almost optical matching between PIB and toluene, the UV-Vis spectrum selectively probes the Ag NP shape, modeled by a prolate ellipsoid with an aspect ratio of 1.2. Hence, the two experimental techniques reveal larger asphericity for Ag@PIB NPs, which might suggest anisotropic grafting of Ag NPs along the two main axes. This large asphericity is expected to impact the structure and the physical properties of Ag@PIB NPs bulk films.

\section{SUPPLEMENTARY MATERIAL}

See the supplementary material for the synthesis of olefinterminated PIB and conversion to thiol-terminated PIB, characterization, SEC traces and ${ }^{1} \mathrm{H}$ NMR of PIB-olefin and PIB-SH 
(Figs. S1 and S2), TGA of PVP-Ag (Fig. S3), TGA graphs and table of the obtained supernatant and nanocomposite precipitate PIB-S- $\mathrm{Ag}_{20 \%}$ and PIB-S-Ag ${ }_{65 \%}$ (Fig. S4), depolarization ratio of the depolarized/polarized light scattering intensities for the two Ag-PIB samples in toluene (Fig. S5), and Perrin's equation (Fig. S6).

\section{ACKNOWLEDGMENTS}

G.F. acknowledges financial support by ERC AdG SmartPhon No. 694977. Y.Z. and G.F. acknowledge the Shanxi Province Hundred Talent Project for the collaboration. L.Y. acknowledges MPIP for hospitality.

\section{REFERENCES}

${ }^{1}$ Y. Huang, Y. Zheng, A. Sarkar, Y. Xu, M. Stefik, and B. C. Benicewicz, "Matrixfree polymer nanocomposite thermoplastic elastomers," Macromolecules 50, 4742 (2017).

${ }^{2}$ S. K. Kumar, B. C. Benicewicz, R. A. Vaia, and K. I. Winey, "50th anniversary perspective: Are polymer nanocomposites practical for applications?," Macromolecules 50, 714 (2017).

${ }^{3}$ M. R. Bockstaller, R. A. Mickiewicz, and E. L. Thomas, "Block copolymer nanocomposites: Perspectives for tailored functional materials,” Adv. Mater. 17, 1331 (2005)

${ }^{4}$ S. Narayanan, J. Choi, L. Porter, and M. R. Bockstaller, "Flexible transparent metal/polymer composite materials based on optical resonant laminate structures," ACS Appl. Mater. Interface 5, 4093 (2013).

${ }^{5}$ A. H. Koch, G. Lévêque, S. Harms, K. Jaskiewicz, M. Bernhardt, A. Henkel, C. Sönnichsen, K. Landfester, and G. Fytas, "Surface asymmetry of coated spherical nanoparticles," Nano Lett. 14(7), 4138 (2014).

${ }^{6}$ Y. Cang, A. N. Reuss, J. Lee, J. Yan, J. Zhang, E. Alonso-Redondo, R. Sainidou, P. Rembert, K. Matyjaszewski, M. R. Bockstaller, and G. Fytas, "Thermomechanical properties and glass dynamics of polymer-tethered colloidal particles and films," Macromolecules 50, 8658 (2017).

${ }^{\mathbf{7}}$ E. Alonso-Redondo, L. Belliard, K. Rolle, B. Graczykowski, W. Tremel, B. Djafari-Rouhani, and G. Fytas, "Robustness of elastic properties in polymer nanocomposite films examined over the full volume fraction range," Sci. Rep. 8, 16986 (2018)

${ }^{8}$ D. S. Reig, P. Hummel, Z. Wang, S. Rosenfeldt, B. Graczykowski, M. Retsch, and G. Fytas, "Well-defined metal-polymer nanocomposites: The interplay of structure, thermoplasmonics, and elastic mechanical properties," Phys. Rev. Mater. 2, 123605 (2018).

9J. Midya, Y. Cang, S. A. Egorov, K. Matyjaszewski, M. R. Bockstaller, A. Nikoubashman, and G. Fytas, "Disentangling the role of chain conformation on the mechanics of polymer tethered particle materials," Nano Lett. 19, 2715 (2019).

${ }^{10}$ M. Gkikas, J. Timonen, J. Ruokolainen, P. Alexandridis, and H. latrou, "Facile aqueous synthesis and stabilization of nearly monodispersed gold nanospheres by poly(L-proline)," J. Polym. Sci. Part A 51, 1448 (2013).

${ }^{11}$ M. Gkikas, G. Theodosopoulos, B. P. Das, M. Tsianou, H. Iatrou, and G. Sakellariou, "Gold-decorating graphene nanosheets composed of a biocompatible non-charged water-soluble polypeptide," Eur. Polym. J. 60, 106 (2014).

${ }^{12}$ M. Gkikas, "Metallic particle assemblies on graphene," Curr. Org. Chem. 19, 1773 (2015)

${ }^{13} \mathrm{M}$. Gkikas, "Graphene-based materials in health and environment: New paradigms," in Hybrid Graphene Metallic Nanoparticles for Biodetection, edited by G. Goncalves, P. Marques, and V. Mercedes (Springer, New York, 2016).

${ }^{14}$ M. Getzin, J. Garfield, D. S. Rundle, U. Krueger, M. Gkikas, and G. J. Wang, "Increased separability of K-edge nanoparticles by photon-counting detectors for spectral $\mu \mathrm{CT}$,” J. X-ray Sci. Technol. 26, 707 (2018).
${ }^{15}$ K. Smith, M. Getzin, J. J. Garfield, S. Suvarnapathaki, G. Camci-Unal, G. Wang, and M. Gkikas, "Nanophosphor-based contrast agents for spectral X-ray imaging,” Nanomaterials 9, 1092 (2019).

${ }^{16}$ S. Ehlert, C. Stegelmeier, D. Pirner, and S. Förster, "A general route to optically transparent highly filled polymer nanocomposites," Macromolecules 48, 5323 (2015).

${ }^{17}$ C.-G. Chao, M. P. Kumar, N. Riaz, R. T. Khanoyan, S. T. Madrahimov, and D. E. Bergbreiter, "Polyisobutylene oligomers as tools for iron oxide nanoparticle solubilization," Macromolecules 50, 1494 (2017).

${ }^{18}$ R. Faust and J. P. Kennedy, "Living carbocationic polymerization. IV. Living polymerization of isobutylene," J. Polym. Sci. Part A 25, 1847 (1987).

${ }^{19}$ Y. C. Bae, Z. Fodor, and R. Faust, "Living coupling reaction in living cationic polymerization: Coupling reaction of living polyisobutylene," Macromolecules 30, 198 (1997).

${ }^{20}$ C. G. Campbell and R. F. Storey, "Carbocationic copolymerization of isobutylene and 2,4-dimethyl-1,3-pentadiene," Macromolecules 51, 6430 (2018).

${ }^{\mathbf{2 1}}$ W. H. Binder, R. Zirbs, D. Machl, and M. Gahleitner, "Grafting polyisobutylene from nanoparticle surfaces: Concentration and surface effects on livingness," Macromolecules 42, 7379 (2009).

${ }^{\mathbf{2 2}}$ D. Pirner, M. Dulle, and S. Förster, "Viscoelastic properties and reinforcement of non-aggregated and aggregated nanocomposites," Polymer 145, 101 (2018).

${ }^{23}$ J. M. D. Lane and G. S. Grest, "Spontaneous asymmetry of coated spherical nanoparticles in solution and at liquid-vapor interfaces," Phys. Rev. Lett. 104, 235501 (2010).

${ }^{24}$ B. Bozorgui, D. Meng, S. K. Kumar, C. Chakravarty, and A. Cacciuto, "Fluctuation-driven anisotropic assembly in nanoscale systems," Nano Lett. 13, 2732 (2013).

${ }^{25}$ D. Zhao, D. Schneider, G. Fytas, and S. K. Kumar, "Controlling the thermomechanical behavior of nanoparticle/polymer films," ACS Nano 8, 8163 (2014).

${ }^{26}$ D. Zhao, V. Gimenez-Pinto, A. M. Jimenez, L. Zhao, J. Jestin, S. K. Kumar, B. Kuei, E. D. Gomez, A. S. Prasad, L. S. Schadler, M. M. Khani, and B. C. Benicewicz, "Tunable multiscale nanoparticle ordering by polymer crystallization,” ACS Cent. Sci. 7, 751 (2017).

${ }^{27}$ P. Akcora, H. Liu, S. K. Kumar, J. Moll, Y. Li, B. C. Benicewicz, L. S. Schadler, D. Acehan, A. Z. Panagiotopoulos, V. Pryamitsyn, V. Ganesan, J. Ilavsky, P. Thiyagarajan, R. H. Colby, and J. F. Douglas, "Anisotropic self-assembly of spherical polymer-grafted nanoparticles,” Nat. Mater. 8, 354 (2009).

${ }^{28}$ A. Girard, H. Gehan, A. Crut, A. Mermet, L. Saviot, and J. Margueritat, "Mechanical coupling in gold nanoparticles supermolecules revealed by plasmon-enhanced ultralow frequency Raman spectroscopy," Nano Lett. 16, 3843 (2016).

${ }^{29}$ K. M. Koczkur, S. Mourdikoudis, L. Polavarapu, and S. E. Skrabalak, "Polyvinylpyrrolidone (PVP) in nanoparticle synthesis," Dalton Trans. 44(41), 17883 (2015).

${ }^{30} \mathrm{P}$. Hummel, L. Lerch, S. M. Goller, M. Karg, and M. Retsch, "Simple and high yield synthesis of metal-polymer nanocomposites: The role of thetacentrifugation as an essential purification step," Polymers 9, 659 (2017).

${ }^{31}$ S. Ehlert, S. M. Taheri, D. Pirner, M. Drechsler, H. W. Schmidt, and S. Förster, "Polymer ligand exchange to control stabilization and compatibilization of nanocrystals," ACS Nano 8, 6114 (2014).

${ }^{32}$ D. N. Benoit, H. Zhu, M. H. Lilierose, R. A. Verm, N. Ali, A. N. Morrison, J. D. Fortner, C. Avendano, and V. L. Colvin, "Measuring the grafting density of nanoparticles in solution by analytical ultracentrifugation and total organic carbon analysis," Anal Chem. 84, 9238 (2012).

${ }^{33}$ P. Voudouris, A. Larsen, B. Loppinet, D. Vlassopoulos, I. Pastoriza-Santos, and L. M. Liz-Marzán, "Static and dynamic plasmon-enhanced light scattering from dispersions of polymer-grafted silver nanoprisms in the bulk and near solid surfaces," J. Phys. Chem. C 116, 3888 (2012).

${ }^{34}$ M. Haghighi, M. A. Plum, G. Gantzounis, H. J. Butt, W. Steffen, and G. Fytas, "Plasmon-enhanced dynamic depolarized light scattering," J. Phys. Chem. C 117, 8411 (2013).

${ }^{35}$ R. Piazza and V. Degiorgio, "Single-particle dynamics in a colloidal crystal," Phys. Rev. Lett. 67, 3868 (1991). 
${ }^{36}$ K. M. Salerno, A. E. Ismail, J. M. Lane, and G. S. Grest, "Coating thickness and coverage effects on the forces between silica nanoparticles in water," J. Chem. Phys. Lett. 140, 194904 (2014).

${ }^{37}$ B. Gao, G. Arya, and A. R. Tao, "Self-orienting nanocubes for the assembly of plasmonic nanojunctions," Nat. Nanotechnol. 7, 433 (2012).

${ }^{38}$ H. Koerner, L. F. Drummy, B. Benicewicz, Y. Li, and R. A. Vaia, "Nonisotropic self-organization of single-component hairy nanoparticle assemblies," ACS Macro Lett. 2, 670 (2013).

${ }^{39}$ J. R. Schmidt and J. L. Skinner, "Brownian motion of a rough sphere and the Stokes-Einstein law," J. Phys. Chem. B 108, 6767 (2004).

${ }^{40}$ G. H. Koenderink, H. Y. Zhang, D. G. A. L. Aarts, M. P. Lettinga, A. P. Philipse, and G. Nagele, "On the validity of Stokes-Einstein-Debye relations for rotational diffusion in colloidal suspensions," Faraday Discuss. 123, 335 (2003).
${ }^{41}$ B. W. M. Kuipers, M. C. A. van de Ven, R. J. Baars, and A. P. Philipse, "Simultaneous measurement of rotational and translational diffusion of anisotropic colloids with a new integrated setup for fluorescence recovery after photobleaching," J. Phys. Condens. Matter 24, 245101 (2012).

${ }^{42}$ A. Tcherniak, S. Dominguez-Medina, W.-S. Chang, P. Swanglap, L. S. Slaughter, C. F. Landes, and S. Link, "One-photon plasmon luminescence and its application to correlation spectroscopy as a probe for rotational and translational dynamics of gold nanorods," J. Phys. Chem. C 115, 15938 (2011).

${ }^{43} \mathrm{~K}$. Wang, X. Qiu, C. Dong, and J. Ren, "Single-molecule technology for rapid detection of DNA hybridization based on resonance light scattering of gold nanoparticles," Chem. Bio. Chem. 8, 1126 (2007).

${ }^{44}$ K. Jaskiewicz, A. Larsen, D. Schaeffel, K. Koynov, I. Lieberwirth, G. Fytas, K. Landfester, and A. Kroeger, "Incorporation of nanoparticles into polymersomes: Size and concentration effects," ACS Nano 6, 7254 (2012). 